\title{
Ictiofauna dos trechos alto e médio da bacia do rio Tibagi, Paraná, Brasil
}

\author{
Oscar Akio Shibatta ${ }^{1,3}$, Ana Maria Gealh ${ }^{2} \&$ Sirlei Terezinha Bennemann ${ }^{1}$ \\ Biota Neotropica v7 (n2) - http://www.biotaneotropica.org.br/v7n2/pt/abstract?article+bn02107022007 \\ Recebido em 26/02/07 \\ Versão reformulada recebida em 04/05/07 \\ Publicado em 28/05/07 \\ ${ }^{1}$ Departamento de Biologia Animal e Vegetal, Universidade Estadual de Londrina, \\ Rod. Celso Garcia Cid, s/n, Campus Universitário, CEP 86051-990, Londrina, PR, Brasil, \\ e-mail: sirlei@uel.br \\ ${ }^{2}$ Departamento de Biologia Geral, Universidade Estadual de Ponta Grossa, Praça Santos Andrade, s/n, \\ CEP 84010-970, Ponta Grossa, PR, Brasil, e-mail: anagealh@uol.com.br \\ ${ }^{3}$ Autor para correspondência: Oscar Akio Shibatta, e-mail: shibatta@uel.br
}

\begin{abstract}
Shibatta, O.A., Gealh, A.M. \& Bennemann, S.T. Ichthyofauna from the middle and upper stretches of rio Tibagi basin, Paraná, Brazil. Biota Neotrop. May/Aug 2007 vol. 7, no. 2. http://www.biotaneotropica.org. br/v7n2/pt/abstract?article+bn02107022007. ISSN 1676-0603.

The ichthyofauna of four stretches of rio Tibagi hydrographic basin was monthly sampled from May 2001 to April 2002, with gillnets (totalling $1,000 \mathrm{~m}^{2}$ ) in order to analyze the dominance, constancy and similarity indexes. A total of 68 species (32 Characiformes, 32 Siluriformes, 2 Gymnotiformes and 2 Perciformes) and 2,758 individuals were captured. The stretch with greatest richness of species was Tibagi abaixo (50 species), followed by Tibagi acima (40 species), Iapó (35) and Fortaleza (14). In Fortaleza occurred the smallest number of species, but the greatest abundance was verified with 508 of 877 captured individuals of Astyanax paranae, what elevated the place's dominance index to $37.9 \%$. The ten more abundant species corresponded to $65.5 \%$ of the total specimens collected, presenting a proportion that varied from 3.9 to $18.6 \%$. From the greatest to smallest percentage, the species were A. paranae, Astyanax fasciatus, Prochilodus lineatus, Hypostomus sp. I, Hypostomus regani, Rhamdia quelen, Astyanax eigenmanniorum, Apareiodon ibitiensis, Apareiodon affinis and Leporinus amblyrhynchus. In frequency of occurrence, 25 species were captured in only one of the stretches; 11 in two stretches; 25 in three; and only R. quelen, A. ibitiensis and A. affinis were captured in all of the stretches. Many migratory species were captured, with highlight to $P$. lineatus and Salminus brasiliensis. The ichthyofauna of Fortaleza, Iapó, Tibagi acima and Tibagi abaixo differed significantly in richness of species, indicating that the fish assemblages are different among stretches. From similarity analysis of Bray-Curtis among stretches, the greatest similarity was verified among Tibagi acima and Tibagi abaixo, followed by Iapó and, finally, Fortaleza. On the other hand, in similarity analyses of Jaccard, the greatest similarity was among Iapó and Tibagi acima, followed by Tibagi abaixo and Fortaleza.

Keywords: conservation, icththyofauna, rivers, rio Tibagi basin, upper rio Paraná.

Resumo

Shibatta, O.A., Gealh, A.M. \& Bennemann, S.T. Ictiofauna dos trechos alto e médio da bacia do rio Tibagi, Paraná, Brasil. Biota Neotrop. May/Aug 2007 vol. 7, no. 2. http://www.biotaneotropica.org.br/v7n2/ pt/abstract?article+bn02107022007. ISSN 1676-0603.

A ictiofauna de quatro trechos da bacia hidrográfica do rio Tibagi foi amostrada mensalmente de maio de 2001 a abril de 2002, com redes de espera (totalizando $1.000 \mathrm{~m}^{2}$ ) para analisar a dominância, constância e similaridade. Um total de 68 espécies (32 Characiformes, 32 Siluriformes, 2 Gymnotiformes e 2 Perciformes) e 2.758 exemplares foram capturados. O trecho com maior riqueza de espécies foi Tibagi abaixo (50 espécies), seguido por Tibagi acima (40 espécies), Iapó (35) e Fortaleza (14). No Fortaleza ocorreu o menor número de espécies, mas foi verificada a maior abundância, com 508 dos 877 exemplares capturados pertencentes à espécie Astyanax paranae, o que elevou o índice de dominância desse local para 37,9\%. As dez espécies mais abundantes corresponderam a 65,5\% do total de exemplares coletados, apresentando uma proporção que variou de 3,9 a 18,6\%. Do maior para o menor percentual, as espécies foram A. paranae, Astyanax fasciatus, Prochilodus lineatus, Hypostomus sp. I, Hypostomus regani, Rhamdia quelen, Astyanax eigenmanniorum, Apareiodon ibitiensis, Apareiodon affinis e Leporinus amblyrhynchus. Quanto à freqüência de ocorrência, 25 espécies foram capturadas em apenas um dos trechos; 11 em dois trechos; 25 em três; e apenas $R$. quelen, A. ibitiensis e A. affinis foram capturadas em todos os trechos. Muitas espécies migratórias foram capturadas, destacando-se P. lineatus e Salminus brasiliensis. A ictiofauna de Fortaleza, Iapó, Tibagi acima e Tibagi abaixo diferiu significativamente em riqueza de espécies, indicando que as assembléias de peixes são diferentes entre
\end{abstract}


os trechos. Da análise de similaridade de Bray-Curtis, verificou-se maior similaridade entre Tibagi acima e Tibagi abaixo, seguido por Iapó e, finalmente, Fortaleza. Já pela análise de similaridade de Jaccard, a maior similaridade foi entre Iapó e Tibagi acima, depois Tibagi abaixo e Fortaleza.

Palavras-chave: conservação, alto rio Paraná, ictiofauna, rios, bacia do rio Tibagi.

\section{Introdução}

O rio Tibagi é um dos poucos rios da região Sul que vêm sendo sistematicamente estudados desde a década de 1980 (Bennemann et al. 1995, Bennemann \& Shibatta 2002, Shibatta et al. 2002). É um rio que ainda apresenta fortes corredeiras e corre encaixado em diversos trechos, embora seja semi-lótico em sua porção inferior devido à influência do reservatório da UHE Escola de Engenharia Mackenzie. Possui grande importância na manutenção da diversidade de peixes da região média da bacia do rio Paranapanema (Hoffmann et al. 2005), que foi reduzida em razão da construção de vários reservatórios ao longo desse trecho.

O Tibagi corre de sul para o norte e tem, aproximadamente 550 km de extensão; nasce no município de Palmeira, nos Campos Gerais, e deságua no município de Sertaneja, ambos no estado do Paraná. Forma a terceira maior bacia desse estado, e pode ser dividida em três regiões conforme o relevo, a hidrologia e a climatologia (De França 2002, Mendonça \& Danni-Oliveira 2002). Na região alta encontram-se algumas das suas áreas mais preservadas, como o Parque Estadual do Guartelá, no rio Iapó, região dos Campos Gerais. Na região média, área de encontro de vários tipos vegetacionais, estão os melhores fragmentos de vegetação nativa da bacia (Torezan 2002). Em função disso, provavelmente, essa região é a mais rica não só em espécies de peixes, mas de espécies em geral, tanto vegetais quanto animais.

Apesar do melhor estado de conservação dessas regiões, no município de Telêmaco Borba está instalada a Usina Hidrelétrica Presidente Vargas com queda de aproximadamente $40 \mathrm{~m}$. Nessa usina foi construído um sistema de transposição de peixes (escada), cuja eficiência tem sido questionada pela população local. A reclamação mais freqüente é a de que não ocorrem mais dourados (Salminus brasiliensis) na região a montante da escada.

Há muito tempo, mas de forma mais intensa atualmente, a bacia do Tibagi é objeto de discussões para a implantação de grandes usinas hidrelétricas. Além disso, ao longo do Tibagi a poluição tem aumentado, o que tem trazido conseqüências diretas sobre a redução da fauna em geral, especialmente a de peixes. Dessa forma, é urgente que as regiões média e alta, ainda pouco estudadas, sejam investigadas. Portanto, este trabalho teve por objetivo descrever e comparar a ictiofauna de quatro trechos em um gradiente longitudinal das regiões média e alta da bacia do rio Tibagi para subsidiar as discussões futuras sobre planos de uso e monitoramento de toda a bacia.

\section{Material e Métodos}

\section{1. Área de Estudo}

As coletas foram realizadas em quatro trechos: médio rio Fortaleza, baixo rio Iapó, e dois trechos do rio Tibagi, um situado acima do Salto Mauá e um abaixo, aqui denominados como Tibagi acima e Tibagi abaixo, respectivamente (Figura 1). O último trecho é o que representa a porção média do rio Tibagi, enquanto que os outros, a região alta.

O Fortaleza é um afluente da margem direita do rio Iapó. Apresenta-se encaixado e com muitos saltos formando cachoeiras e regiões de corredeiras, mas com suas margens em estado de franca erosão. Seu fundo é rochoso (arenitos devonianos da Formação Furnas), com presença de areia de granulação média a grossa. O trecho Fortaleza (Figura 2a), localizado na fazenda Santo Amaro, no município de Tibagi ( $50^{\circ} 20^{\prime} \mathrm{S}$ e $\left.24^{\circ} 40^{\prime} \mathrm{W}\right)$, tem vegetação ripária formada por um remanescente de floresta ombrófila mista, com elementos de savana gramíneo-lenhosa; essa vegetação é cercada por campos úmidos e brejosos nativos (primários), por campos rochosos e pedregosos com elementos de cerrado, e está em bom estado de preservação. Nas imediações o solo é utilizado, principalmente, para o cultivo de soja, milho e feijão, e também para a agropecuária.

O Iapó é um dos principais afluentes da margem direita do rio Tibagi. Apresenta-se encaixado, com regiões de corredeiras entremeadas por locais de amplas várzeas pantanosas. Seu fundo é rochoso (arenitos devonianos da Formação Furnas e diques de diabásio) e areno-argiloso. O trecho Iapó (Figura 2b) localiza-se a jusante do canyon do Guartelá, na fazenda Capão Grande, município de Tibagi $\left(50^{\circ} 01^{\prime} \mathrm{S}\right.$ e $\left.24^{\circ} 51^{\prime} \mathrm{W}\right)$, na região alta a vegetação ripária é formada por um remanescente de floresta ombrófila mista e encontra-se em adiantado estágio de regeneração. Apresenta macrófitas aquáticas em abundância. A área de entorno encontra-se bastante impactada pelas atividades agropecuárias, com pastagens de Brachiaria sp.

O Tibagi é o principal afluente do rio Paranapanema. É conhecido pelo grande número de cachoeiras que apresenta, e a cachoeira do salto Mauá é aproveitada pela Usina Hidrelétrica Presidente Vargas, localizada no município de Telêmaco Borba, implantada na década de 1950. Na barragem dessa usina foi construída uma escada para a subida dos peixes e, por isso, foram estabelecidos dois trechos de coletas no rio Tibagi, o Tibagi acima e o Tibagi abaixo, os quais são separados por essa barragem.

O trecho Tibagi acima (Figura 2c), inserido na região alta, está a montante do salto Mauá e localiza-se no Bairro Conceição, no município de Tibagi (50 $54^{\prime}$ 'S e $\left.24^{\circ} 42^{\prime} \mathrm{W}\right)$. Caracteriza-se por apresentar poucas corredeiras e pequena planície aluvial na margem esquerda. Fundo e margens são predominantemente rochosos (arenitos do Grupo Itararé e diques de diabásio). A mata ripária é formada por um remanescente de floresta ombrófila mista. Na margem esquerda do rio, que já foi um porto de areia, a mata encontra-se degradada, no estágio de capoeirão; há, ainda, abundância de macrófitas aquáticas. Na margem direita há uma reserva florestal da Indústria Klabin S/A, em bom estado de conservação.

O trecho Tibagi abaixo (Figura 2d), a jusante do Salto Mauá, localiza-se na fazenda Recreio Bom Jesus, no Bairro dos Felisberto, no município de Curiúva $\left(50^{\circ} 70^{\prime} \mathrm{S}\right.$ e $\left.24^{\circ} 02^{\prime} \mathrm{W}\right)$, região média da bacia. É cercado por morros e apresenta muitas corredeiras com águas rasas e poços com até $50 \mathrm{~m}$ de profundidade. O fundo é rochoso (arenito da Formação Rio do Rasto e diques de diabásio). A vegetação ripária é remanescente de floresta estacional semidecidual submontana. Esse tipo de vegetação está condicionado pela dupla estacionalidade climática: uma tropical, com intensas chuvas de verão seguidas por estiagens acentuadas, e outra subtropical sem período seco, mas com seca fisiológica provocada pelo intenso frio de inverno. Constitui-se de macrofanerófitos que revestem solos basálticos eutróficos, com elementos comuns à floresta ombrófila atlântica. A mata ripária constitui uma associação secundária em equilíbrio. Nas margens encachoeiradas há moitas de esponjinha e sarandi, com ingá-mirim nas anfractuosidades. O entorno mostra-se impactado 


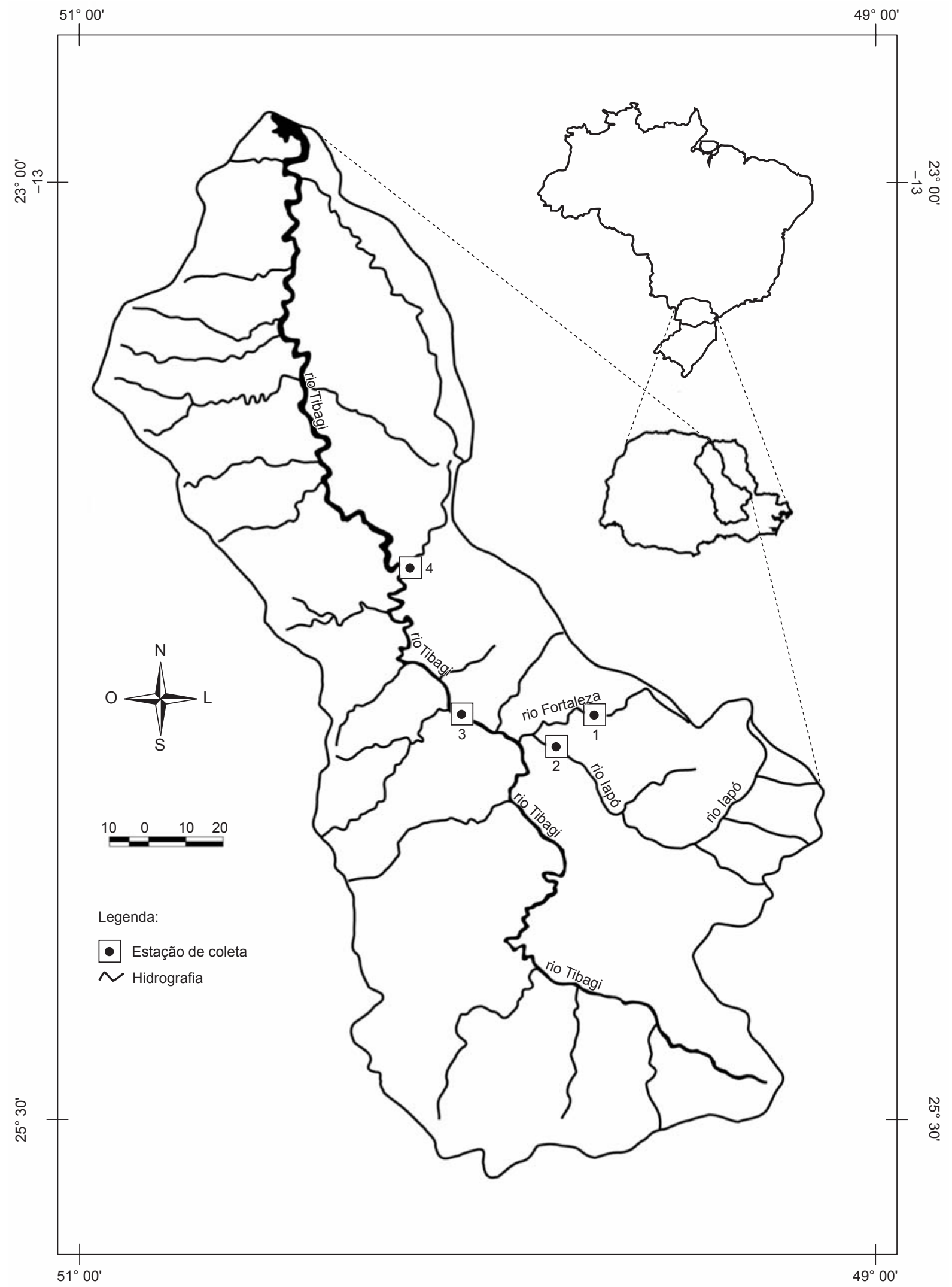

Figura 1. Localização dos trechos de coleta na bacia do rio Tibagi: 1 = Fortaleza; 2 = Iapó; 3 = Tibagi acima; 4 = Tibagi abaixo.

Figure 1. Localization of collection stretches in the rio Tibagi basin: 1 = Fortaleza; 2 = Iapó; 3 = Tibagi acima; $4=$ Tibagi abaixo. 


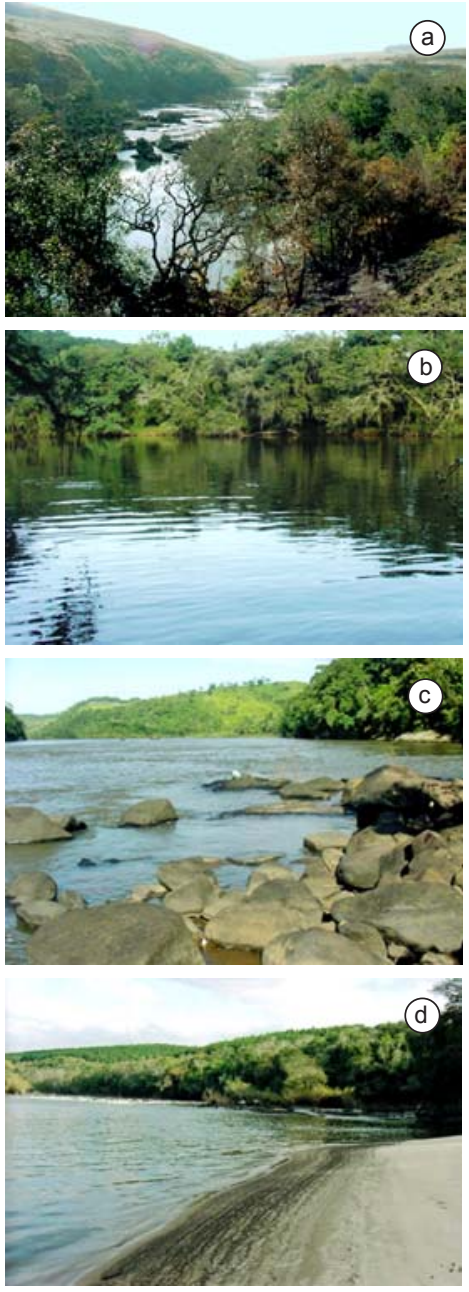

Figura 2. Trechos de coletas. a) Fortaleza, localizado na fazenda Santo Amaro, município de Tibagi; b) Iapó, localizado a jusante do canyon do Guartelá, na fazenda Capão Grande, município de Tibagi; c) Tibagi acima, montante do Salto Mauá, localizado no bairro Conceição, município de Tibagi; e d) Tibagi abaixo, jusante do salto Mauá, localizado na fazenda Recreio Bom Jesus, Município de Curiúva.

Figure 2. Collection stretches. a) Fortaleza, localized in Santo Amaro farmer, Tibagi municipality; b) Iapó, localized downstream of Guartelá canyon, in Capão Grande farmer, Tibagi municipality; c) Tibagi acima, localized upstream of Salto Mauá, localized in bairro Conceição, Tibagi municipality; and d) Tibagi abaixo, downstream of salto Mauá, localized in Recreio Bom Jesus farmer, Curiúva municipality.

pela atividade agropecuária, inclusive nos cumes dos morros, e por áreas de reflorestamento próximas.

\section{Amostragem e análise de dados}

As capturas dos peixes foram realizadas mensalmente no período de maio de 2001 a abril de 2002. O esforço de pesca aplicado foi de $1.000 \mathrm{~m}^{2}$ de rede de espera com malhas $2,4,6,8,10,12$ e $14 \mathrm{~cm}$ entre nós adjacentes e $15 \mathrm{~m}$ de comprimento, durante períodos de 24 horas, com despescas a cada 12 horas (noturno e diurno). Para as tarrafas, de malha 4, foram estipulados 20 lances diurnos (às 12 horas) e 20 noturnos (às 22 horas), para todas as coletas e em cada trecho.

Os índices de dominância, assim como a similaridade entre os trechos (índice de Bray-Curtis e Jaccard) foram calculados com o software PAST (Hammer et al. 2003). A constância foi calculada segundo Dajoz (1983). Foram consideradas constantes as espécies presentes em mais de $50 \%$ das coletas; acessórias as que estiveram presentes entre 25 a $50 \%$, inclusive; e acidentais as que ocorreram em menos de $25 \%$ das coletas.

\section{Resultados}

As capturas totalizaram 2.758 exemplares, distribuídos em 68 espécies de quatro ordens (32 espécies da ordem Characiformes, 32 Siluriformes, 2 Gymnotiformes e 2 Perciformes) (Tabela 1).

$\mathrm{O}$ trecho com maior riqueza de espécies foi o Tibagi abaixo (50 espécies), seguido por Tibagi acima (40 espécies), Iapó (35) e Fortaleza (14). As maiores riquezas de espécies foram observadas nos rios de maior porte, cujo número de espécies aumenta consideravelmente a partir do trecho localizado no rio Fortaleza, o de menor porte, em direção ao trecho Tibagi abaixo.

Das 61 espécies coletadas no rio Tibagi neste estudo, apenas 29 foram comuns aos dois trechos. Apesar do menor número de espécies, Fortaleza foi o trecho que apresentou maior abundância, com 508 dos 877 exemplares capturados pertencentes à espécie A. paranae, o que elevou o índice de dominância desse trecho para 37,9\%. Outros trechos tiveram baixos índices de dominância, com Iapó apresentando 8,5\%, Tibagi acima, 8,0\%, e Tibagi abaixo, $6,8 \%$.

As dez espécies mais abundantes corresponderam a $65,0 \%$ do total, apresentando uma proporção que variou de 3,8 a $18,4 \%$. São elas: Astyanax paranae (18,4\%), Astyanax fasciatus $(7,0 \%)$, Prochilodus lineatus (5,7\%), Hypostomus sp. I (5,6\%), Hypostomus regani $(5,2 \%)$, Rhamdia quelen (5,1\%), Astyanax eigenmanniorum $(4,9 \%)$, Apareiodon ibitiensis $(4,7 \%)$, Apareiodon affinis $(4,6 \%) \mathrm{e}$ Leporinus amblyrhynchus (3,8\%) (Tabela 1).

Da análise de similaridade de Bray-Curtis entre os trechos, verifica-se maior similaridade entre Tibagi acima e Tibagi abaixo, em seguida com Iapó e, finalmente, o menos similar, Fortaleza (Figura 3). Já na análise de Similaridade de Jaccard, a maior similaridade foi entre Iapó e Tibagi acima, depois Tibagi abaixo e, finalmente, Fortaleza (Figura 4).

Em relação à presença das espécies nos trechos de coleta, 26 foram capturadas em apenas um dos trechos, $12 \mathrm{em}$ dois, $25 \mathrm{em}$ três, e apenas $R$. quelen, A. ibitiensis e A. affinis foram capturadas nos quatro trechos. A constância das espécies para cada trecho de coleta está apresentada na Tabela 2. Astyanax fasciatus foi constante no trecho Tibagi acima e Tibagi abaixo, denotando preferência por rios de maior porte; A. altiparanae, no trecho Tibagi abaixo; e A. eigenmanniorum, no Iapó. Astyanax paranae foi constante durante todo o ano no trecho Fortaleza e não foi capturada nos outros, indicando preferência por rios de menor porte (Tabela 2).

Oito espécies migratórias foram coletadas nos trechos Tibagi acima e Tibagi abaixo: Leporinus friderici, L. elongatus, L. obtusidens, L. octofasciatus, Schizodon nasutus, P. lineatus, A. affinis e Iheringichthys labrosus. As espécies Pimelodus maculatus, Parodon tortuosus e Salminus brasiliensis foram coletadas apenas no trecho Tibagi abaixo, e Salminus hilarii apenas no Tibagi acima.

As espécies S. brasiliensis e P. lineatus são migratórias de longas distâncias. Apenas a segunda foi capturada tanto no Tibagi acima quanto no Tibagi abaixo e no Iapó. Poucos exemplares da primeira $(\mathrm{n}=3)$ foram capturados, denotando que é rara na região, enquanto a segunda pode ser considerada comum $(n=157)$.

As corredeiras da região mantêm alta diversidade de cascudos, sendo seis espécies ainda não descritas. As 16 espécies identificadas correspondem à expressiva proporção de $24 \%$ do total de espécies 
Tabela 1. Número de indivíduos das espécies de peixes capturadas em quatro trechos de rios da bacia do rio Tibagi, no período de maio de 2001 a abril de 2002. Cha $=$ Characiformes, Sil $=$ Siluriformes, Gym $=$ Gymnotiformes, Per $=$ Perciformes .

Table 1. Number of individuals of fish species captured in four stretches of rivers from rio Tibagi basin, in the period of May 2001 to April 2002. Cha $=$ Characiformes, Sil $=$ Siluriformes, Gym $=$ Gymnotiformes, Per $=$ Perciformes.

\begin{tabular}{|c|c|c|c|c|c|c|c|}
\hline Espécies & Ordem & Fortaleza & Iapó & $\begin{array}{l}\text { Tibagi } \\
\text { acima }\end{array}$ & $\begin{array}{c}\text { Tibagi } \\
\text { abaixo }\end{array}$ & Total & $\begin{array}{c}\text { Proporção } \\
(\%)\end{array}$ \\
\hline Apareiodon affinis & Cha & 2 & 3 & 24 & 98 & 127 & 4,60 \\
\hline Apareiodon ibitiensis & Cha & 105 & 11 & 7 & 6 & 129 & 4,68 \\
\hline Apareiodon piracicabae & Cha & 0 & 16 & 45 & 35 & 96 & 3,48 \\
\hline Astyanax altiparanae & Cha & 0 & 2 & 3 & 30 & 35 & 1,27 \\
\hline Astyanax eigenmanniorum & Cha & 4 & 108 & 23 & 0 & 135 & 4,89 \\
\hline Astyanax fasciatus & Cha & 0 & 27 & 89 & 77 & 193 & 7,00 \\
\hline Astyanax paranae & Cha & 508 & 0 & 0 & 0 & 508 & 18,42 \\
\hline Brycon nattereri & Cha & 0 & 14 & 2 & 0 & 16 & 0,58 \\
\hline Bryconamericus iheringii & Cha & 1 & 0 & 2 & 1 & 4 & 0,15 \\
\hline Bryconamericus stramineus & Cha & 0 & 0 & 1 & 0 & 1 & 0,04 \\
\hline Corydoras ehrhardti & Sil & 0 & 0 & 6 & 2 & 8 & 0,29 \\
\hline Corydoras paleatus & Sil & 0 & 0 & 0 & 3 & 3 & 0,11 \\
\hline Crenicichla haroldoi & Per & 1 & 0 & 0 & 5 & 6 & 0,22 \\
\hline Cyphocharax modestus & Cha & 0 & 0 & 1 & 0 & 1 & 0,04 \\
\hline Eigenmannia trilineata & Gym & 0 & 1 & 0 & 0 & 1 & 0,04 \\
\hline Eigenmania virescens & Sil & 0 & 2 & 4 & 1 & 7 & 0,25 \\
\hline Galeocharax knerii & Cha & 0 & 0 & 0 & 18 & 18 & 0,65 \\
\hline Geophagus brasiliensis & Per & 14 & 10 & 5 & 0 & 29 & 1,05 \\
\hline Gymnotus sylvius & Gym & 0 & 1 & 1 & 0 & 2 & 0,07 \\
\hline Hoplias malabaricus & Cha & 11 & 1 & 4 & 0 & 16 & 0,58 \\
\hline Hypostomus albopunctatus & Sil & 0 & 25 & 5 & 16 & 46 & 1,67 \\
\hline Hypostomus ancistroides & Sil & 0 & 0 & 0 & 3 & 3 & 0,11 \\
\hline Hypostomus hermanni & Sil & 0 & 4 & 10 & 27 & 41 & 1,49 \\
\hline Hypostomus iheringi & Sil & 0 & 66 & 11 & 0 & 77 & 2,79 \\
\hline Hypostomus margaritifer & Sil & 0 & 0 & 8 & 2 & 10 & 0,36 \\
\hline Hypostomus regani & Sil & 0 & 34 & 54 & 54 & 142 & 5,15 \\
\hline Hypostomus sp. I & Sil & 0 & 58 & 87 & 9 & 154 & 5,58 \\
\hline Hypostomus sp. IV & Sil & 0 & 0 & 0 & 5 & 5 & 0,18 \\
\hline Hypostomus sp. V & Sil & 0 & 10 & 3 & 2 & 15 & 0,54 \\
\hline Hypostomus sp. VI & Sil & 0 & 0 & 0 & 6 & 6 & 0,22 \\
\hline Hypostomus sp. VII & Sil & 0 & 0 & 0 & 2 & 2 & 0,07 \\
\hline Hypostomus strigaticeps & Sil & 0 & 0 & 0 & 2 & 2 & 0,07 \\
\hline Hypostomus variostictus & Sil & 0 & 3 & 0 & 0 & 3 & 0,11 \\
\hline Iheringichthys labrosus & Sil & 0 & 70 & 4 & 13 & 87 & 3,15 \\
\hline Imparfinis schubarti & Sil & 0 & 0 & 2 & 0 & 2 & 0,07 \\
\hline Leporellus vittatus & Cha & 0 & 4 & 7 & 7 & 18 & 0,65 \\
\hline Leporinus amblyrhynchus & Cha & 0 & 58 & 25 & 23 & 106 & 3,84 \\
\hline Leporinus elongatus & Cha & 1 & 0 & 2 & 11 & 14 & 0,51 \\
\hline Leporinus friderici & Cha & 0 & 0 & 1 & 6 & 7 & 0,25 \\
\hline Leporinus obtusidens & Cha & 0 & 1 & 0 & 9 & 10 & 0,36 \\
\hline Leporinus octofasciatus & Cha & 0 & 8 & 16 & 4 & 28 & 1,02 \\
\hline Leporinus striatus & Cha & 0 & 0 & 1 & 12 & 13 & 0,47 \\
\hline Loricaria prolixa & Sil & 0 & 0 & 0 & 13 & 13 & 0,47 \\
\hline Megalancistrus parananus & Sil & 0 & 0 & 0 & 2 & 2 & 0,07 \\
\hline Megalonema platanus & Sil & 0 & 1 & 3 & 9 & 13 & 0,47 \\
\hline Myleus tiete & Cha & 0 & 0 & 0 & 1 & 1 & 0,04 \\
\hline
\end{tabular}


Tabela 1. Continuação..

\begin{tabular}{|c|c|c|c|c|c|c|c|}
\hline Espécies & Ordem & Fortaleza & Iapó & $\begin{array}{l}\text { Tibagi } \\
\text { acima }\end{array}$ & $\begin{array}{c}\text { Tibagi } \\
\text { abaixo }\end{array}$ & Total & $\begin{array}{c}\text { Proporção } \\
(\%)\end{array}$ \\
\hline Neoplecostomus sp. & Sil & 96 & 0 & 0 & 0 & 96 & 3,48 \\
\hline Oligosarcus paranensis & Cha & 0 & 3 & 17 & 4 & 24 & 0,87 \\
\hline Parodon tortuosus & Cha & 0 & 0 & 0 & 27 & 27 & 0,98 \\
\hline Piabina argentea & Cha & 3 & 0 & 0 & 0 & 3 & 0,11 \\
\hline Piaractus mesopotamicus & Cha & 0 & 0 & 2 & 0 & 2 & 0,07 \\
\hline Pimelodus heraldoi & Sil & 0 & 18 & 7 & 20 & 45 & 1,63 \\
\hline Pimelodus maculatus & Sil & 0 & 0 & 0 & 9 & 9 & 0,33 \\
\hline Pimelodus paranensis & Sil & 0 & 1 & 0 & 2 & 3 & 0,11 \\
\hline Prochilodus lineatus & Cha & 0 & 40 & 24 & 93 & 157 & 5,69 \\
\hline Pseudopimelodus mangurus & Sil & 0 & 1 & 2 & 2 & 5 & 0,18 \\
\hline Pseudocetopsis gobioides & Sil & 0 & 0 & 0 & 2 & 2 & 0,07 \\
\hline Rhamdia quelen & Sil & 113 & 11 & 15 & 2 & 141 & 5,11 \\
\hline Salminus hilarii & Cha & 0 & 10 & 9 & 0 & 19 & 0,69 \\
\hline Salminus brasiliensis & Cha & 0 & 0 & 0 & 3 & 3 & 0,11 \\
\hline Schizodon intermedius & Cha & 0 & 0 & 0 & 2 & 2 & 0,07 \\
\hline Schizodon nasutus & Cha & 0 & 1 & 18 & 14 & 33 & 1,20 \\
\hline Serrasalmus maculatus & Cha & 0 & 0 & 0 & 3 & 3 & 0,11 \\
\hline Sorubim lima & Sil & 0 & 0 & 0 & 2 & 2 & 0,07 \\
\hline Steindachneridion scripta & Sil & 0 & 1 & 3 & 2 & 6 & 0,22 \\
\hline Steindachnerina insculpta & Cha & 0 & 1 & 0 & 2 & 3 & 0,11 \\
\hline Trichomycterus diabolus & Sil & 13 & 0 & 0 & 0 & 13 & 0,47 \\
\hline Trichomycterus sp. II & Sil & 5 & 0 & 0 & 0 & 5 & 0,18 \\
\hline Número total de exemplares & - & 877 & 622 & 553 & 703 & 2758 & 100 \\
\hline Número total de espécies & - & 14 & 35 & 40 & 50 & 68 & - \\
\hline
\end{tabular}

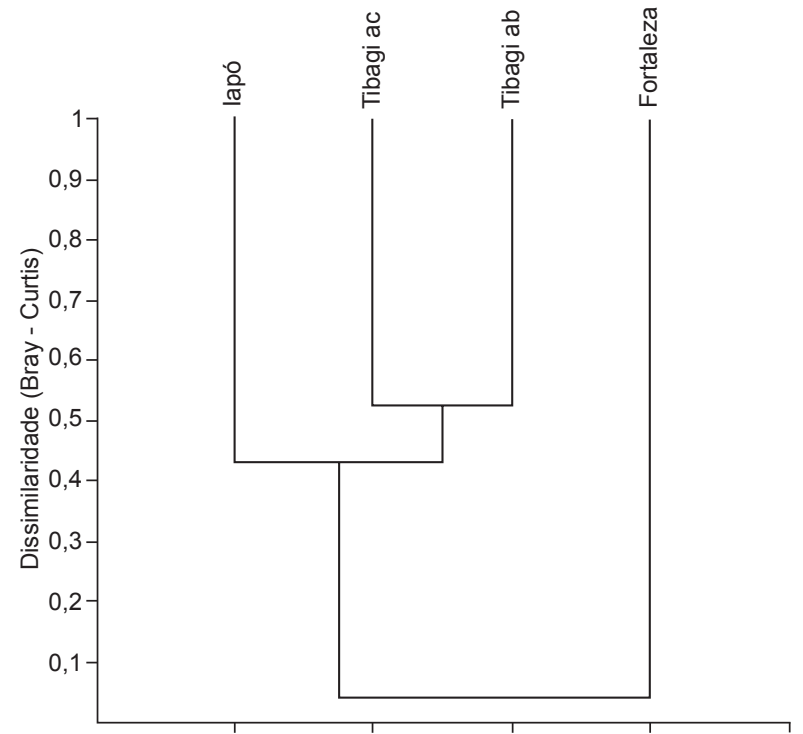

Figura 3. Dendrograma de similaridade (Bray-Curtis) entre os trechos de rios da bacia do rio Tibagi, no período de maio de 2001 a abril de 2002.

Figure 3. Similarity dendrogram (Bray-Curtis) between stretches of rivers from rio Tibagi basin, in the period of May 2001 to April 2002.

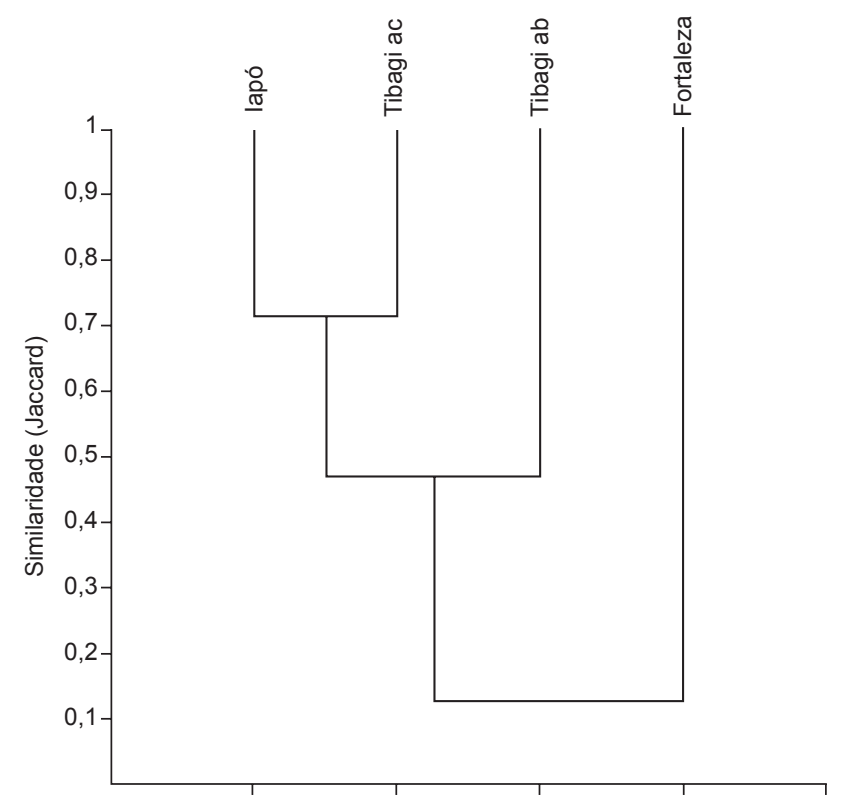

Figura 4. Dendrograma de similaridade (Jaccard) entre os trechos de rios da bacia do rio Tibagi, no período de maio de 2001 a abril de 2002.

Figure 4. Similarity dendrogram (Jaccard) between stretches of rivers from rio Tibagi basin, in the period of May 2001 to April 2002. 
Tabela 2. Constância de captura das espécies de peixes em quatro trechos de rios da bacia do rio Tibagi, no período de maio de 2001 a abril de 2002. $(\mathrm{CT})=$ Constante; $(\mathrm{AS})=$ Acessória; $(\mathrm{AC})=$ Acidental.

Table 2. Capture constance of fish species in four stretches of rivers from rio Tibagi basin, in the period of May 2001 to April 2002 . (CT) = Constant; $(\mathrm{AS})=$ Accessory $;(\mathrm{AC})=$ Accidental.

\begin{tabular}{|c|c|c|c|c|}
\hline Espécie & Fortaleza & Iapó & Tibagi acima & Tibagi abaixo \\
\hline Apareiodon affinis & $16,7(\mathrm{AC})$ & $25,0(\mathrm{AS})$ & $83,3(\mathrm{CT})$ & $66,7(\mathrm{CT})$ \\
\hline Apareiodon ibitiensis & 91,7 (CT) & $33,3(\mathrm{AS})$ & $33,3(\mathrm{AS})$ & $25,0(\mathrm{AS})$ \\
\hline Apareiodon piracicabae & - & $58,3(\mathrm{CT})$ & $83,3(\mathrm{CT})$ & $50,0(\mathrm{AS})$ \\
\hline Astyanax altiparanae & - & $8,3(\mathrm{AC})$ & $25,0(\mathrm{AS})$ & $75,5(\mathrm{CT})$ \\
\hline Astyanax eigenmanniorum & $8,3(\mathrm{AC})$ & $83,3(\mathrm{CT})$ & $50,0(\mathrm{AS})$ & - \\
\hline Astyanax fasciatus & - & $50,0(\mathrm{AS})$ & $91,7(\mathrm{CT})$ & $83,3(\mathrm{CT})$ \\
\hline Astyanax paranae & $100,0(\mathrm{CT})$ & - & - & - \\
\hline Brycon nattereri & - & $50,0(\mathrm{AS})$ & $16,7(\mathrm{AC})$ & - \\
\hline Bryconamericus iheringii & $8,3(\mathrm{AC})$ & - & $8,3(\mathrm{AC})$ & $8,3(\mathrm{AC})$ \\
\hline Bryconamericus stramineus & - & - & $8,3(\mathrm{AC})$ & - \\
\hline Corydoras ehrhardti & - & - & $25,0(\mathrm{AS})$ & $16,7(\mathrm{AC})$ \\
\hline Corydoras paleatus & - & - & - & $16,7(\mathrm{AC})$ \\
\hline Crenicichla haroldoi & $8,3(\mathrm{AC})$ & - & - & $33,3(\mathrm{AS})$ \\
\hline Cyphocharax modestus & - & - & $8,3(\mathrm{AC})$ & - \\
\hline Eigenmannia trilineata & - & $8,3(\mathrm{AC})$ & - & - \\
\hline Eigenmania virescens & - & $8,3(\mathrm{AC})$ & $16,7(\mathrm{AC})$ & $8,3(\mathrm{AC})$ \\
\hline Galeocharax knerii & - & - & - & $83,3(\mathrm{CT})$ \\
\hline Geophagus brasiliensis & $50,0(\mathrm{AS})$ & $41,7(\mathrm{AS})$ & $33,3(\mathrm{AS})$ & - \\
\hline Gymnotus sylvius & - & $8,3(\mathrm{AC})$ & $8,3(\mathrm{AC})$ & - \\
\hline Hoplias malabaricus & $41,7(\mathrm{AS})$ & $8,3(\mathrm{AC})$ & $25,0(\mathrm{AS})$ & - \\
\hline Hypostomus albopunctatus & - & $75,5(\mathrm{CT})$ & $58,3(\mathrm{CT})$ & $25,0(\mathrm{AS})$ \\
\hline Hypostomus ancistroides & - & - & - & $16,7(\mathrm{AC})$ \\
\hline Hypostomus hermanni & - & $16,7(\mathrm{AC})$ & $16,7(\mathrm{AC})$ & $33,3(\mathrm{AS})$ \\
\hline Hypostomus iheringi & - & $33,3(\mathrm{AS})$ & $33,3(\mathrm{AS})$ & - \\
\hline Hypostomus margaritifer & - & - & $25,0(\mathrm{AS})$ & $8,3(\mathrm{AC})$ \\
\hline Hypostomus regani & - & 75,5 (CT) & $83,3(\mathrm{CT})$ & $66,7(\mathrm{CT}) \backslash$ \\
\hline Hypostomus sp. I & - & $66,7(\mathrm{CT})$ & $83,3(\mathrm{CT})$ & $50,00(\mathrm{AS})$ \\
\hline Hypostomus sp. IV & - & - & - & $25,0(\mathrm{AS})$ \\
\hline Hypostomus sp. V & - & $33,3(\mathrm{AS})$ & $25,00(\mathrm{AS})$ & $8,3(\mathrm{AC})$ \\
\hline Hypostomus sp. VI & - & - & - & $16,7(\mathrm{AC})$ \\
\hline Hypostomus sp. VII & - & - & - & $8,3(\mathrm{AC})$ \\
\hline Hypostomus strigaticeps & - & - & - & $8,3(\mathrm{AC})$ \\
\hline Hypostomus variostictus & - & $8,3(\mathrm{AC})$ & - & - \\
\hline Iheringichthys labrosus & - & $75,5(\mathrm{CT})$ & $25,0(\mathrm{AS})$ & $50,0(\mathrm{AS})$ \\
\hline Imparfinis schubarti & - & - & $8,3(\mathrm{AC})$ & - \\
\hline Leporellus vittatus & - & $25,0(\mathrm{AS})$ & $50,0(\mathrm{AS})$ & $41,7(\mathrm{AS})$ \\
\hline Leporinus amblyrhynchus & - & $75,5(\mathrm{CT})$ & $75,5(\mathrm{CT})$ & $58,3(\mathrm{CT})$ \\
\hline Leporinus elongatus & $8,3(\mathrm{AC})$ & - & $8,3(\mathrm{AC})$ & $50,0(\mathrm{AS})$ \\
\hline Leporinus friderici & - & - & $8,3(\mathrm{AC})$ & $25,0(\mathrm{AS})$ \\
\hline Leporinus obtusidens & - & $8,3(\mathrm{AC})$ & - & $33,3(\mathrm{AS})$ \\
\hline Leporinus octofasciatus & - & $50,0(\mathrm{AS})$ & $75,5(\mathrm{CT})$ & $33,3(\mathrm{AS})$ \\
\hline Leporinus striatus & - & - & $8,3(\mathrm{AC})$ & $58,3(\mathrm{CT})$ \\
\hline Loricaria prolixa & - & - & - & $33,3(\mathrm{AS})$ \\
\hline Megalancistrus parananus & - & - & - & $16,7(\mathrm{AC})$ \\
\hline Megalonema platanus & - & $8,3(\mathrm{AC})$ & $25,0(\mathrm{AS})$ & $50,0(\mathrm{AS})$ \\
\hline Myleus tiete & - & - & - & $8,3(\mathrm{AC})$ \\
\hline Neoplecostomus sp. & $100,0(\mathrm{CT})$ & - & - & - \\
\hline Oligosarcus paranensis & - & $16,7(\mathrm{AC})$ & $50,0(\mathrm{AS})$ & $25,0(\mathrm{AS})$ \\
\hline Parodon tortuosus & - & - & - & $50,0(\mathrm{AS})$ \\
\hline
\end{tabular}


Tabela 2. Continuação...

\begin{tabular}{|c|c|c|c|c|}
\hline Espécie & Fortaleza & Iapó & Tibagi acima & Tibagi abaixo \\
\hline Piabina argentea & $8,3(\mathrm{AC})$ & - & - & - \\
\hline Piaractus mesopotamicus & - & - & $8,3(\mathrm{AC})$ & - \\
\hline Pimelodus heraldoi & - & $66,7(\mathrm{CT})$ & $50,0(\mathrm{AS})$ & 75,0 (CT) \\
\hline Pimelodus maculatus & - & - & - & $58,3(\mathrm{CT})$ \\
\hline Pimelodus paranensis & - & $8,3(\mathrm{AC})$ & - & $16,7(\mathrm{AC})$ \\
\hline Prochilodus lineatus & - & $75,5(\mathrm{CT})$ & $75,5(\mathrm{CT})$ & $100,0(\mathrm{CT})$ \\
\hline Pseudopimelodus mangurus & - & $8,3(\mathrm{AC})$ & $16,7(\mathrm{AC})$ & $16,7(\mathrm{AC})$ \\
\hline Pseudocetopsis gobioides & - & - & - & $16,7(\mathrm{AC})$ \\
\hline Rhamdia quelen & $100,0(\mathrm{CT})$ & $41,7(\mathrm{AS})$ & $58,3(\mathrm{CT})$ & $16,7(\mathrm{AC})$ \\
\hline Salminus hilarii & - & $58,3(\mathrm{CT})$ & $41,7(\mathrm{AS})$ & - \\
\hline Salminus brasiliensis & - & - & - & $16,7(\mathrm{AC})$ \\
\hline Schizodon intermedius & - & - & - & $16,7(\mathrm{AC})$ \\
\hline Schizodon nasutus & - & $8,3(\mathrm{AC})$ & $75,5(\mathrm{CT})$ & $66,7(\mathrm{CT})$ \\
\hline Serrasalmus maculatus & - & - & - & $25,0(\mathrm{AS})$ \\
\hline Sorubim lima & - & - & - & $8,3(\mathrm{AC})$ \\
\hline Steindachneridion scripta & - & $8,3(\mathrm{AC})$ & $25,0(\mathrm{AS})$ & $16,7(\mathrm{AC})$ \\
\hline Steindachnerina insculpta & - & $8,3(\mathrm{AC})$ & - & $16,7(\mathrm{AC})$ \\
\hline Trichomycterus diabolus & $33,3(\mathrm{AS})$ & - & - & - \\
\hline Trichomycterus sp. II & $8,3(\mathrm{AC})$ & - & - & - \\
\hline Percentagem de espécies em cada categoria & $\begin{aligned} \mathrm{CT} & =29 \% \\
\mathrm{AC} & =50 \% \\
\mathrm{AS} & =21 \%\end{aligned}$ & $\begin{aligned} \mathrm{CT} & =29,5 \% \\
\mathrm{AC} & =41 \% \\
\mathrm{AS} & =29,5 \%\end{aligned}$ & $\begin{aligned} \mathrm{CT} & =28 \% \\
\mathrm{AC} & =34 \% \\
\mathrm{AS} & =38 \%\end{aligned}$ & $\begin{aligned} \mathrm{CT} & =22 \% \\
\mathrm{AC} & =42 \% \\
\mathrm{AS} & =36 \%\end{aligned}$ \\
\hline
\end{tabular}

encontradas na região (68). Além dessas, uma das duas espécies de Trichomycterus, com distribuição restrita ao rio Fortaleza, é nova para a ciência.

As espécies Brycon nattereri, Myleus tiete e Steindachneridion scripta são raras e encontram-se na listas de espécies ameaçadas de extinção no Brasil. É notável a ocorrência de seis espécies do gênero Leporinus no rio Tibagi, e algumas delas também foram capturadas nos rios Iapó e Fortaleza.

Analisando-se o gênero Apareiodon, constata-se que A. affinis tem preferência por rios de maior porte, tendo sido constante no rio Tibagi. Apareiodon piracicabae foi constante nos rios Iapó e Tibagi e A. ibitiensis apenas no rio Fortaleza, significando preferência por rios menores. A espécie carnívora $H$. malabaricus ocorreu principalmente no rio Fortaleza (onde foi coletada nos poções).

As espécies capturadas apenas no trecho Tibagi acima foram Bryconamericus stramineus, Imparfinis schubarti e Piaractus mesopotamicus. Apenas Hypostomus variostictus foi exclusiva do rio Iapó, e A. eigenmanniorum, B. nattereri, L. amblyrhynchus, I. labrosus, Hypostomus iheringi e H. albopunctatus mostraram preferência por esse rio, como pode ser observado pela maior abundância de exemplares (Tabela 1).

\section{Discussão}

A maior proporção de espécies das ordens Characiformes e Siluriformes encontrada nas regiões alta e média da bacia do rio Tibagi segue uma tendência observada nos rios da região neotropical, como verificado no Estado de São Paulo (Castro \& Menezes 1998), na Amazônia (Lowe-McConnell 1999), no Pantanal (Britski et al. 1999) e também na bacia do rio Tibagi como um todo (Shibatta et al. 2002). Entretanto, no presente estudo estiveram ausentes as ordens Cyprinodontiformes e Synbranchiformes, provavelmente devido ao método de coleta utilizado, que não permitiu capturar, por exemplo, exemplares de Phalloceros caudimaculatus nem de Synbranchus marmoratus que ocorrem na bacia do rio Tibagi. A utilização de coletas padronizadas com redes de espera com malhas superiores a dois centímetros entre nós adjacentes também pode ter provocado uma sub-estimativa do número de espécies pequenas, principalmente no que concerne ao rio Fortaleza, uma vez que essas são mais comuns em rios de menor porte. Outros métodos de captura como redes de arrasto e peneiras podem revelar outras espécies de pequeno porte, aumentando o número de espécies para essas regiões.

Na região alta da bacia do rio Tibagi, Bennemann et al. (1995), em um estudo sazonal, registraram 15 espécies num trecho localizado no município de Ipiranga. No presente estudo mais 51 espécies foram registradas para essa região. Esses resultados confirmam a necessidade de maior freqüência de coletas, assim como amostragens em maior número de afluentes, para se obter um quadro mais completo sobre a diversidade da fauna de peixes em determinada região de uma bacia.

Para a bacia do rio Tibagi, com este estudo, são acrescentadas mais 13 espécies além das 110 citadas por Shibatta et al. (2002). Além disso, dez não puderam ser identificadas, podendo representar espécies novas. Na lista de espécies não descritas destacam-se os cascudos, que vivem em ambientes de corredeiras e podem ser utilizadas como representativas desse tipo de ambiente. Somando-se todas as espécies de cascudos identificadas neste estudo, atinge-se o total de 16 espécies, o que corresponde a $64 \%$ do número de espécies apontadas por Jerep (2006) para a bacia do rio Tibagi (25 espécies). Algumas dessas espécies, no entanto, são diferentes entre os dois estudos, o que representa um aumento da riqueza desses peixes para a bacia. Outra espécie não descrita, pertencente ao gênero Trichomycterus, foi capturada no rio Fortaleza, e também apresenta adaptações à vida 
em corredeiras e em meio ao substrato rochoso. Portanto, antes da implantação de empreendimentos que tragam alterações das condições naturais de qualquer trecho do rio Tibagi ou de seus tributários, deve haver investigação com maior abrangência temporal e espacial, com a finalidade de se conhecer a riqueza da comunidade de peixes em profundidade.

Foram registradas duas espécies alóctones à bacia, P. mesopotamicus e Sorubim lima, provenientes de escapes acidentais de pisciculturas ou de introduções intencionais. Ao longo da bacia do rio Tibagi existem várias estações de piscicultura que podem contribuir com introduções de espécies, como observado por Orsi \& Agostinho (1999) na região do baixo Tibagi, que registraram o escape de milhões de exemplares de várias espécies de peixes numa época de chuvas intensas, quando os tanques transbordaram.

Dentre as espécies ameaçadas de extinção, foram registradas neste estudo S. scripta, B. nattereri e M. tiete. A captura de 12 exemplares de $S$. scripta evidencia que a espécie é rara, provavelmente por atingir grande porte e por ser carnívora. A presença das espécies herbívoras Brycon nattereri e $M$. tiete, que dependem dos recursos que a mata ripária pode oferecer (folhas, flores e frutos), indica que as matas ainda estão relativamente bem preservadas nesses trechos, apesar dos diversos impactos ambientais existentes na bacia Entretanto, como ambas foram pouco abundantes, essa preservação já não é suficiente para sustentar populações numerosas.

Destaca-se a riqueza de espécies congêneres, principalmente dos gêneros Astyanax, Leporinus, Apareiodon e Hypostomus, que coexistem nos rios estudados. Considerando esses grupos e suas características biológicas particulares, associadas à diversidade de hábitat que assegura a sua sobrevivência, entende-se a necessidade da preservação dos diferentes ambientes em um ecossistema, para que a biodiversidade seja assegurada. Além disso, existem poucos estudos sobre a coexistência de várias espécies de peixes de água doce neotropicais do mesmo gênero. As do gênero Astyanax são algumas das mais conhecidas na literatura sob os aspectos da alimentação (Arcifa et al. 1991, Esteves \& Galetti, 1995, Vilella et al. 2002). Bennemann et al. (2005) observaram, nos mesmos trechos e períodos aqui analisados, que A. altiparanae, A. fasciatus, A. paranae (identificado como A. aff. scabripinnis) e A. eigenmanniorum utilizam a área marginal, embora tenham sido constantes em trechos diferentes. Além disso, A. eigemanniorum destacou-se quanto à estratégia alimentar, pois, em vez de consumir alimentos alóctones provenientes das margens, utilizou plantas aquáticas (Bryophyta) que ficavam aderidas às rochas das áreas marginais, em locais de maior profundidade.

No presente estudo, seis espécies de Leporinus foram capturadas no mesmo trecho (Tibagi abaixo), mas com apenas L. amblyrhynchus, ocorrendo constantemente em todos os trechos, exceto no Fortaleza. Possivelmente esta espécie se segrega ao utilizar de maneira diferenciada os recursos e aproveitar a abundância de algum alimento em especial, que provavelmente devem ser as plantas aderidas nas rochas, pois entre as seis, é a que tem a boca localizada mais ventralmente. Bennemann et al. (2000) verificaram segregação temporal em três espécies que utilizavam essencialmente os mesmos recursos alimentares num trecho da região baixa do rio Tibagi. Balassa et al. (2004) analisaram a dieta de quatro espécies desse gênero e observaram diferenças morfológicas e segregação espacial. Portanto, os fatores que provavelmente permitem a coexistência das espécies de Leporinus são as diferenças no tamanho do corpo, do padrão de colorido e da dentição, que possibilitam a utilização diferenciada de alimentos e de ocupação do espaço físico.

As três espécies da família Parodontidae foram coletadas em trechos com características lóticas, de fundo rochoso. A alimentação dessas espécies foi analisada e os resultados (A. M. Gealh, dados não publicados) foram similares, com consumo principalmente de briófitas que se aderem às rochas (e uma diversidade de larvas de insetos aquáticos que utilizam essas plantas como substrato). Um comportamento semelhante deve ocorrer com as espécies do gênero Hypostomus que necessitam dos ambientes de corredeiras e dos alimentos que ficam aderidos às rochas, os quais elas raspam. Isso deve explicar a sua grande diversidade nos rios Iapó e Tibagi, nos quais foram registradas em maior número de espécies e de exemplares. A presença dessas espécies que utilizam o perifito como alimento indica que o ambiente tem sofrido pouco assoreamento, uma vez que esses organismos microscópicos seriam soterrados com o acúmulo de sedimentos. O baixo acúmulo de sedimento pode estar relacionado com a alta velocidade da água e não com a ausência de degradação da mata ciliar, uma vez que algumas espécies mais sensíveis, como B. nattereri, altamente dependente dos recursos alimentares oferecidos pela vegetação marginal, não foram registradas em abundância nos trechos amostrados.

A riqueza de espécies foi diferente entre os trechos acima e abaixo da barragem da Usina Hidrelétrica Presidente Vargas (UHEPV). Entretanto, a influência do sistema de transposição de peixes da UHEPV (STP-UHEPV) sobre a migração dos peixes deve ser analisada com cautela, mesmo que diferenças na composição de espécies migratórias entre as áreas a montante e à jusante dessa barragem pudessem indicar impedimento à migração. Pimelodus maculatus, P. tortuosus e $S$. brasiliensis só ocorreram no trecho Tibagi abaixo e $S$. hilarii no Tibagi acima. Somente três exemplares de Salminus brasiliensis (dourado) foram coletados durante os 12 meses de amostragens, e apenas no trecho Tibagi abaixo, evidenciando que é uma espécie rara e que pode estar ameaçada de extinção na bacia, principalmente se as grandes barragens projetadas forem construídas. Mesmo assim, a raridade de $S$. brasiliensis acima dela pode não estar relacionada somente à instalação da UHEPV, pois é uma espécie carnívora que normalmente não se encontra em grande abundância nos ambientes. Além disso, dentre as 16 espécies migratórias registradas por Shibatta et al. (2002) para o rio Tibagi, oito foram coletadas nos trechos Tibagi acima e Tibagi abaixo. Por exemplo, Prochilodus lineatus destacase por ter sido capturada em abundância e constância tanto acima quanto abaixo da usina. Gealh-Escobar (1991) e Moraes (1995) também verificaram a presença de Prochilodus lineatus na região alta do rio Tibagi (represa Alagados, rio Pitangui e Lagoa Dourada). A sua presença pode significar que, pelo menos para ela, o sistema de transposição pode estar sendo utilizado para a migração.

O Tibagi ainda mantém características que possibilitam a manutenção de algumas espécies migratórias, que conseguem completar o seu ciclo de vida mesmo com o STP-UHEPV. Seria importante que estudos mais aprofundados sobre a migração de peixes sejam realizados, incluindo a marcação e recaptura das espécies, utilização de marcadores moleculares, além da localização de seus sítios de reprodução. Além disso, um monitoramento eficiente é recomendável para que os efeitos de qualquer alteração ambiental de maior amplitude sejam identificados e acompanhados, permitindo tomar decisões que amenizem ao máximo os impactos causados. Assim, além de estudar por um maior período e abrangência de trechos e tributários, é importante preservar as características físicas, impedindo-se a instalação de obstáculos como, por exemplo, grandes barragens e fontes poluidoras, que atingirão diretamente essas espécies.

\section{Agradecimentos}

Ao Mário Sérgio de Melo, coordenador do projeto Caracterização do Patrimônio Natural dos Campos Gerais do Paraná. Ao Cláudio Henrique Zawadzki, pela identificação das espécies de Hypostomus. Aos pescadores profissionais que nos auxiliaram nas coletas de campo e a Urias Bueno de Camargo e Tereza Antunes Texeira de Camargo, por 
permitirem a montagem do laboratório de campo em sua propriedade. Ao apoio de Karin Vangela de Geus e família. A Igor Fabiano Nazar, pelo trabalho de computação. Ao Marco Aurélio Fonseca Santos, pela responsabilidade em nos conduzir para Tibagi. Este trabalho teve apoio da Prefeitura Municipal de Tibagi, do CNPq (520992/99-7), da Fundação Araucária (002/2001, protoc. 596) e da UEPG.

\section{Referências Bibliográficas}

ARCIFA, M.S., NORTHCOTE, T.G. \& FROEHLICH, O. 1991. Interactive ecology of two cohabiting characin (Astyanax fasciatus and Astyanax bimaculatus) in an eutrophic Brasilian reservoir. J. Trop. Ecology 7:257-268

BALASSA, G.C., FUGI, R., HAHN, N.S. \& GALINA, A.B. 2004. Dieta de espécies de Anostomidae (Teleostei, Characiformes) na área de influência do reservatório de Manso, Mato Grosso, Brasil. Iheringia, Sér. Zool. 94:77-82.

BENNEMANN, S.T., GEALH, A.M., ORSI, M.L. \& SOUZA, L.M. 2005. Ocorrência e ecologia trófica de quatro espécies de Astyanax (Characidae) em diferentes rios da bacia do rio Tibagi, Paraná, Brasil. Iheringia, Sér. Zool. 95:247-254.

BENNEMANN, S.T. \& SHIBATTA, O.A. 2002. Dinâmica de uma assembléia de peixes do rio Tibagi. In A bacia do rio Tibagi (M.E. Medri, E. Bianchini, O.A. Shibatta \& J.A. Pimenta, eds.). M. E. Medri, Londrina, p.433-442.

BENNEMANN, S.T., SHIBATTA, O.A. \& GARAVELLO, J.C. 2000. Peixes do rio Tibagi: uma abordagem ecológica. Eduel, Londrina.

BENNEMANN, S.T., SILVA-SOUZA, A.T. \& ROCHA, G.R.A. 1995. Composición ictiofaunistica em cinco localidades de la cuenca Del rio Tibagi, PR - Brasil. Interciencia 20:7-13.

BRITSKI, H.A., SILIMON, K.Z.S. \& LOPES, B.S. 1999. Peixes do Pantanal. Manual de identificação. Embrapa, Brasília.

CASTRO, R.M.C. \& MENEZES, N.A. 1998. Estudo diagnóstico da diversidade de peixes do estado de São Paulo. In Biodiversidade do estado de São Paulo, Brasil: síntese do conhecimento ao final do século XX, 6: vertebrados. R. M. C. Castro (ed.), São Paulo, FAPESP. p. 3-13.

DAJOZ, R. 1983. Ecologia geral, 4 ed. Vozes, São Paulo.

DE FRANÇA, V. 2002. o rio Tibagi no contexto hidrográfico parananense. In A bacia do rio Tibagi (M.E. Medri, E. Bianchini, O.A. Shibatta \& J.A. Pimenta, eds.). M. E. Medri, Londrina, p.45-61.
ESTEVES, K.E. \& GALETTI, P.M. 1995. Food partitioning among characids of a small Brazilian floodplain lake from the Paraná River basin. Environ. Biol. Fishes 42:375-389.

GEALH-ESCOBAR, A.M. 1991. Aspectos da biologia e da estrutura de comunidade de peixes da Represa Alagados, Ponta Grossa, PR. Dissertação de Mestrado, Universidade Federal do Paraná, Curitiba.

HAMMER, O., HARPER, D.A. \& RYAN, P.D. 2003. PAST - Paleontolological Statistics ver. 1.12. (Disponível em http://www.folk.uio.no/ohammer/ past.) Acessado em: 31/07/2003.

HOFFMANN, A.C., ORSI, M.L. \& SHIBATTA, O.A. 2005. Diversidade de peixes do reservatório da UHE Escola Engenharia Mackenzie (Capivara), Rio Paranapanema. Bacia do alto rio Paraná, Brasil, e a importância dos grandes tributários na sua manutenção. Iheringia, Sér. Zool. 95 (3): 319-325.

JEREP, F.C. 2006. Cascudos (Loricariidae: Siluriformes) da bacia do médio e baixo rio Paranapanema, alto Paraná, Brasil. Dissertação de mestrado, Universidade Estadual de Londrina, Londrina.

LOWE-McCONNELL, R.H. 1999. Estudo ecológico de comunidades de peixes tropicais. Edusp, São Paulo.

MENDONÇA, F.A. \& DANNI-OLIVEIRA, I.M. 2002. Dinâmica atmosférica e tipos climáticos predominantes da bacia do rio Tibagi. In A bacia do rio Tibagi (M.E. Medri, E. Bianchini, O.A. Shibatta \& J.A. Pimenta, eds.). Londrina, M. E. Medri, p.63-66.

MORAES, M.F.P.G. 1995. Estudo do tubo digestivo e do hábito alimentar de Hoplias malabaricus, Geophagus brasiliensis e Prochilodus lineatus (Osteichthyes, Teleostei) da Lagoa Dourada, Ponta Grossa, Paraná. Dissertação de Mestrado, Universidade Federal do Paraná, Curitiba.

ORSI, M.L. \& AGOSTINHO, A.A. 1999. Introdução de espécies de peixes por escapes acidentais de tanques de cultivo em rios da bacia do rio Paraná, Brasil. Revta. Bras. Zool. 16:557-560.

SHIBATTA, O.A., ORSI, M.L., BENNEMANN, S.T. \& SILVA-SOUZA, A.T. 2002. Diversidade e distribuição de peixes na bacia do rio Tibagi. In A bacia do rio Tibagi (M.E. Medri, E. Bianchini, O.A. Shibatta \& J.A. Pimenta, eds.). Londrina, M. E. Medri, p.403-423.

TOREZAN, J.M.D. 2002. Nota sobre a vegetação da bacia do rio Tibagi. In A bacia do rio Tibagi (M.E. Medri, E. Bianchini, O.A. Shibatta \& J.A. Pimenta, eds.). Londrina, M. E. Medri, p.103-107.

VILELLA, F.S., BECKER, F.G. \& HARTZ, S.M. 2002. Diet of Astyanax species (Teleostei, Characidae) in a Atlantic Forest River in Southern Brazil. Braz. Arch. Biol. Technol. 45:223-232. 